\title{
PAPER
}

\section{Melissa officinalis extract in the treatment of patients with mild to moderate Alzheimer's disease: a double blind, randomised, placebo controlled trial}

\author{
*S Akhondzadeh, M Noroozian, M Mohammadi, S Ohadinia, A H Jamshidi, M Khani
}

See end of article for

J Neurol Neurosurg Psychiatry 2003;74:863-866

authors' affiliations

Correspondence: $\mathrm{Dr}$

Shahin Akhondzadeh,

Associate Professor,

Roozbeh Psychiatric

Hospital, Tehran University

of Medical Sciences, South

Kargar Avenue, Tehran

13334, Iran;

s.akhond@neda.net

Received

8 November 2002

In revised form

18 January 2003

Accepted 9 March 2003

*The roles in the study of the coauthors are given in the appendix

\begin{abstract}
Objective: To assess the efficacy and safety of Melissa officinalis extract using a fixed dose 160 drops/day) in patients with mild to moderate Alzheimer's disease.

Design: A four month, parallel group, placebo controlled trial undertaken in three centres in Tehran, Iran.

Methods: Patients with mild to moderate Alzheimer's disease aged between 65 and 80 years $(n=42$; 18 women, 24 men) with a score of $\geqslant 12$ on the cognitive subscale of Alzheimer's disease assessment scale (ADAS-cog) and $\leqslant 2$ on the clinical dementia rating (CDR) were randomised to placebo or fixed dose of Melissa officinalis extract. The main efficacy measures were the change in the ADAS-cog and CDR-SB scores compared with baseline. Side effects were systematically recorded.

Results: At four months, Melissa officinalis extract produced a significantly better outcome on cognitive function than placebo (ADAS-cog: $d f=1, F=6.93, p=0.01 ; C D R: d f=1, F=16.87$, $p<0.0001)$. There were no significant differences in the two groups in terms of observed side effects except agitation, which was more common in the placebo group $(p=0.03)$.

Conclusions: Melissa officinalis extract is of value in the management of mild to moderate Alzheimer's disease and has a positive effect on agitation in such patients.
\end{abstract}

A zheimer's disease is the leading cause of dementia in mid to late life and is estimated to affect approximately 20 million people worldwide. There are considerable financial, social, and emotional costs associated with the burden of caring for patients with this disease..$^{1-3}$ The first neurotransmitter defect discovered in Alzheimer's disease involved acetylcholine. As cholinergic function is required for short term memory, the cholinergic deficit in Alzheimer's disease was also believed to be responsible for much of the short term memory deficit. ${ }^{45}$ Clinical drug trials in patients with Alzheimer's disease have focused on drugs that augment levels of acetylcholine in the brain to compensate for the loss of cholinergic function. These drugs have included acetylcholine precursors, muscarinic agonists, nicotinic agonists, and acetylcholine esterase inhibitors. The most highly developed and successful approaches to date have employed acetylcholine esterase inhibition. ${ }^{6}$ Pharmacological treatment strategies in Alzheimer's disease include three classes of agents: mechanism based disease modifying treatments such as vitamin E and selegiline; mechanism based treatments that compensate for transmitter deficits such as acetylcholine esterase inhibitors; and psychotropic agents given to relieve the behavioural symptoms of Alzheimer's disease. ${ }^{7-10}$

Although two Food and Drug Administration approved drugs are available to neurologists for the management of Alzheimer's disease, the outcomes are not very promising and there is a place for alternative drugs and in particular for phytotherapy. In this respect, herbal medicines may be a new avenue of treatment for Alzheimer's disease. ${ }^{3610}$ It has been suggested-on the basis of a retrospective review of the historical role of various European herbs in the improvement of cognition, and in particular memory-that Melissa officinalis and another herb in the labiatae family, Salvia officinalis, might provide a natural treatment for Alzheimer's disease. ${ }^{11}{ }^{12}$ Melissa officinalis comes from Europe and is now grown all over the world. As well as having soothing and carminative effects, it also has anxiolytic and sedative/hypnotic actions. ${ }^{13}$ It has been reported that Melissa officinalis has acetylcholine receptor activity in the central nervous system, with both nicotinic and muscarinic binding properties. ${ }^{14}$ Moreover, a recent study showed that this herb modulates mood and cognitive performance during acute administration in healthy young volunteers. ${ }^{15}$ No side effects or symptoms of toxicity have been reported with its use. ${ }^{13-15}$

We undertook the present study to test the efficacy and safety of a fixed dose of Melissa officinalis over a four month period in patients with mild to moderate Alzheimer's disease, using a double blind, randomised, placebo controlled trial design.

\section{METHODS}

\section{Trial organisation}

This was a four month, parallel group, placebo controlled trial undertaken in three centres in Tehran, Iran, during October 2000 to September 2002. Overall coordination of the trial was from the Institute of Medicinal Plants, Tehran.

\section{Participants}

Eligible participants in the study were male and female outpatients aged between 65 and 80 years. Patients with a history of cognitive decline that had been gradual in onset and progressive for at least six months were included. Other inclusion criteria were:

Abbreviations: ADAS-cog, cognitive subscale of the Alzheimer's disease assessment scale; CDR, clinical dementia rating; CDR-SB, clinical dementia rating-sum of the boxes; LOCF, last observation carried forward procedure; OC, observed cases 


\begin{tabular}{|c|c|c|c|}
\hline & Melissa extract & Placebo & $\mathrm{p}$ Value \\
\hline $\begin{array}{l}\text { Age lyears) } \\
\text { (mean (SD)) }\end{array}$ & $73(3.8)$ & 73.7 (3.7) & 0.54 \\
\hline Sex & $M 12 ; F 9$ & M 12; F 9 & 1.24 \\
\hline
\end{tabular}

- A diagnosis of probable Alzheimer's disease according to the criteria of National Institute of Neurological and Communicative Disorders and Stroke and Alzheimer's Disease and Related Disorders Association (NINCDS/ADRDA). ${ }^{16}$

- The presence of mild to moderate dementia (score of $\geqslant 12$ on the cognitive subscale of Alzheimer's disease assessment scale (ADAS-cog) and $\leqslant 2$ on the clinical dementia rating scale $(\mathrm{CDR}) .{ }^{17} 18$

Patients also had to have regular contact with a responsible caregiver. Those with concomitant diseases such as hypertension, congestive heart failure, non-insulin-dependent diabetes mellitus, and hypothyroidism were included in the study provided the disease was controlled. Patients were excluded from the study if they had evidence of other neurodegenerative disorders; any cardiovascular disease likely to prevent completion of the study; clinically significant psychiatric diseases; urinary outflow obstruction; an active peptic ulcer; any history of epilepsy; or significant drug or alcohol misuse. Any other drugs being taken to treat dementia had to be discontinued. The use of other concomitant drug treatment was permitted, except that, where possible, drugs with a psychotropic action were discontinued 48 hours before cognitive evaluation. Drugs with anticholinergic effects or cholinomimetic effects were avoided.

ADAS-cog is a subscale of 11 items that evaluates selected aspects of attention, language, memory, orientation, praxis, and reasoning. Scores for ADAS-cog range from 0 to 70 (very severe). ${ }^{17}$ The clinical dementia rating-sum of the boxes (CDR-SB) score provides a consensus based global clinical measure by summing the ratings from six domains: memory, orientation, judgment, problem solving, community affairs, home and hobbies, and personal care. Ratings are assigned by the function in relation to cognitive ability and past performance, with an increase in score denoting deterioration. ${ }^{18}$ Patients were randomised to receive Melissa officinalis extract or placebo, 60 drops/day, in a $1: 1$ ratio using a computer generated code. The assignments were kept in sealed, opaque envelopes until the point of allocation.

The trial was carried out in accordance with the Declaration of Helsinki and subsequent revisions, and was approved by the ethics committee at the National Research Centre of Medical Sciences of Iran. The patient (or a representative), together with the carer, provided written informed consent for participation.

\section{Interventions}

Patients were randomised to receive Melissa officinalis extract 60 drops/day or placebo 60 drops/day. Throughout the study the person who administered the preparations, the rater, and the patients were blind to the assignments.

\section{Extract preparation}

Melissa officinalis (leaf) was obtained from the farm of the Institute of Medicinal Plants, Halejerd, Iran. The taxonomic identity of the plants was confirmed by the botanist of the Department of Cultivation and Development of Institute of Medicinal Plants, Tehran. The plant extract was prepared as $1: 1$ in $45 \%$ alcohol. The extract was standardised to contain at least $500 \mu \mathrm{g} \mathrm{citral} / \mathrm{ml}$.

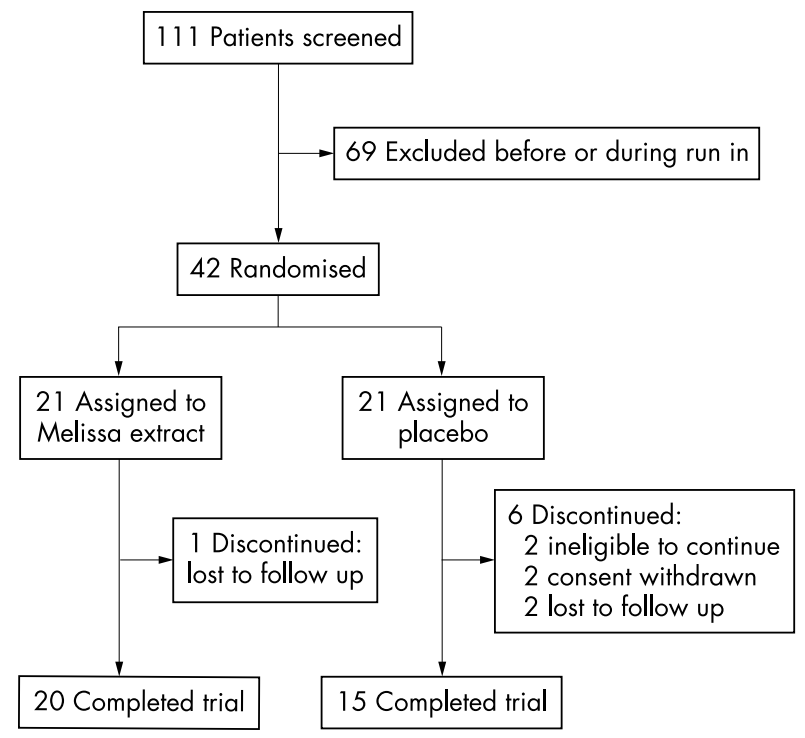

Figure 1 Trial profile.

\section{Outcomes}

The main efficacy measures were the ADAS-cog and CDR-SB. Outcome measures were the change in ADAS-cog and CDR-SB scores over the trial. Patients were assessed by a neurologist at baseline and every two weeks after the treatment started.

\section{Safety evaluation}

All adverse events, reported or observed, were recorded at each visit. Routine physical examinations were conducted at each clinic visit. Complete physical examinations, including 12 lead ECG recordings, were conducted at week 0 , week 8 , and week 16.

\section{Statistical analysis}

Using data from a pilot study and considering a five point difference in change in the ADAS-cog score between patients treated with Melissa and placebo, we calculated that we needed at least 15 patients in each arm. A two way repeated measures analysis of variance (time-treatment interaction) was used. We considered the two groups as the between-subjects factor (group) and the nine measurements during treatment as the within-subjects factor (time). This was done for both ADAS-cog and CDR-SB scores. In addition, a one way repeated measures analysis of variance with a two tailed post hoc Tukey mean comparison test was undertaken on the change in ADAS-cog and CDR-SB from baseline. To compare the reduction in score of the ADAS-cog and CDR-SB scales at week 16 in relation to baseline, an unpaired two sided Student's $t$ test was used. Fisher's exact test was employed to compare the baseline data and frequency of side effects between the protocols. Results are presented as mean (SEM) and were considered significant at a probability $(\mathrm{p})$ value of $\leqslant 0.05$. A traditional "observed cases" (OC) analysis (the patients who completed the trial) at 16 weeks was the primary efficacy analysis. In addition, intention to treat analysis with the last observation carried forward (LOCF) procedure was also done. All results discussed are based on OC analysis unless otherwise stated.

\section{RESULTS}

One hundred and eleven patients were screened for the study and 42 were randomised to the trial preparations ( 21 patients in each group). No significant differences were identified between patients randomly assigned to the Melissa extract group or the placebo group with regard to basic demographic data including age and sex (table 1). Thirty five patients completed the trial. One patient in the Melissa extract group and six in the placebo group dropped out. Although the number of dropouts in the 


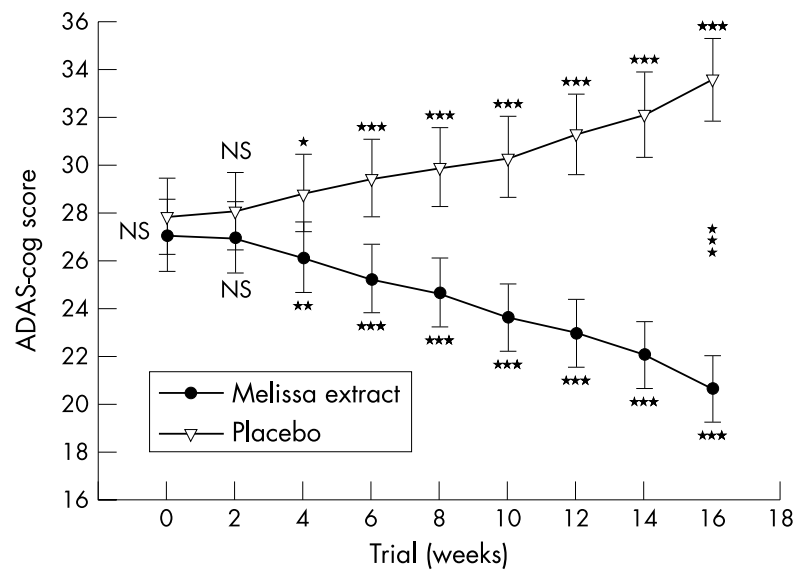

Figure 2 Mean scores of the two groups on the cognitive subscale of the Alzheimer's disease assessment scale (ADAS-cog). Error bars $=$ SEM. ${ }^{*}<0.05 ;{ }^{* *}<0.01 ;{ }^{* * *}<0.001$.

placebo group was higher than in the Melissa extract group, the difference was not statistically significant $(\mathrm{p}=0.09)$ (fig 1$)$.

\section{Efficacy \\ ADAS-cog}

The mean (SEM) scores of two groups of patients are shown in fig 2. There were no significant differences between the two groups at baseline (week 0) on the ADAS-cog rating scale $(t=0.36, \mathrm{df}=33, \mathrm{p}=0.71)$. The difference between the two preparations was significant as indicated by the effect of group (between-subjects factor): $\mathrm{F}=6.93, \mathrm{df}=1, \mathrm{p}=0.01$, and $\mathrm{F}=5.95, \mathrm{df}=1, \mathrm{p}=0.01$, for OC and LOCF analyses, respectively. The behaviour of the two treatments was not homogeneous across time (group by time interaction, GreenhouseGeisser correction: $\mathrm{F}=242.78, \mathrm{df}=3.09, \mathrm{p}<0.0001)$. In addition, a one way repeated measures analysis of variance showed a significant effect of both protocols on the ADAS-cog rating scale scores $(p<0.0001)$.

In both groups post hoc comparisons showed a significant change from week 4 on the ADAS-cog rating scale scores (fig 2 ). The difference between the two protocols was significant at the end point (week 16) $(t=5.76, \mathrm{df}=33, \mathrm{p}<0.0001$, and $t=5.26, \mathrm{df}=40, \mathrm{p}<0.0001$ for OC and LOCF analyses, respectively). The changes at the end point compared with baseline were (mean (SD)): -6.40 (1.66) and 5.60 (1.40) for Melissa extract and placebo, respectively. A significant difference was observed on the change of scores of the ADAS-cog rating scale at week 16 compared with baseline in the two groups $(t=22.50, \mathrm{df}=33, \mathrm{p}<0.0001$, and $t=16.50$, $\mathrm{df}=40, \mathrm{p}<0.0001$ for OC and LOCF analyses, respectively).

\section{CDR-SB}

The mean (SEM) scores of two groups of patients are shown in fig 3. There were no significant differences between two groups at baseline (week 0 ) on the CDR-SB $(t=0.15, \mathrm{df}=33$, $\mathrm{p}=0.87)$. The difference between the two preparations was significant as indicated by the effect of group (betweensubjects factor): $\mathrm{F}=16.87, \mathrm{df}=1, \mathrm{p}<0.0001$, and $\mathrm{F}=23.92$, $\mathrm{df}=1, \mathrm{p}<0.0001$ for OC and LOCF analyses, respectively. The behaviour of the two treatments was not homogeneous across the time (group by time interaction: $\mathrm{F}=40.75, \mathrm{df}=8$, $\mathrm{p}<0.0001)$. In addition, a one way repeated measures analysis of variance showed a significant effect of both protocols on the CDR-SB scores $(p<0.0001)$.

In both groups, post hoc comparisons showed a significant change from week 8 on the CDR-SB scores. The difference between the two protocols was significant at the end point (week 16) $(t=7.45, \mathrm{df}=33, \mathrm{p}<0.0001$, and $t=8.25$, $\mathrm{df}=40, \mathrm{p}<0.0001$ for OC and LOCF analyses, respectively).

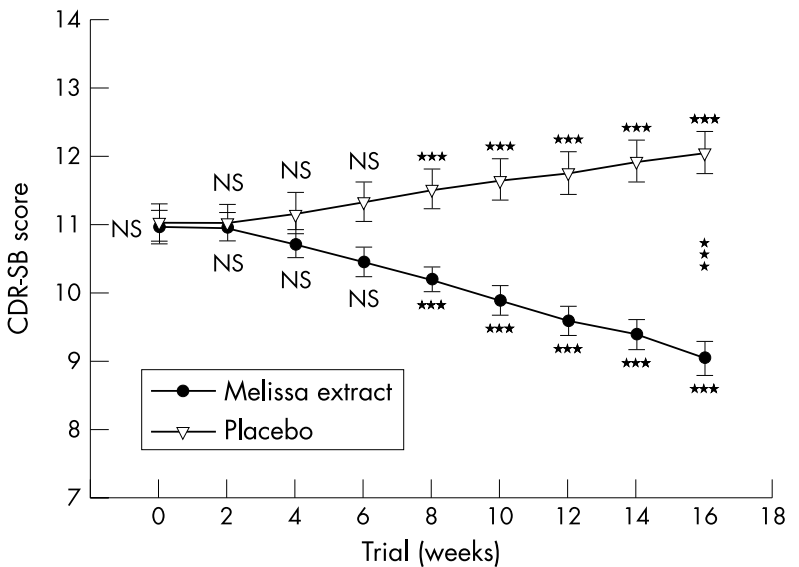

Figure 3 Mean scores of the two groups on the clinical dementia rating-sum of the boxes scale (CDR-SB). Error bars $=$ SEM. ${ }^{* *}<$ $0.01 ; * * *<0.001$.

\begin{tabular}{llll}
\hline Table 2 & Number of patients with side effects \\
\hline Side effect & Melissa extract & Placebo & p Value \\
\hline Vomiting & 3 & 1 & 1.00 \\
Dizziness & 1 & 1 & 1.39 \\
Wheezing & 2 & 0 & 0.10 \\
Agitation & 1 & 6 & 0.03 \\
Abdominal pain & 2 & 0 & 0.48 \\
Nausea & 1 & 0 & 0.48 \\
\hline
\end{tabular}

The changes at the end point compared to baseline were (mean (SD)): -1.92 (1.48) and 1.03 (0.54) for Melissa extract and placebo, respectively. A significant difference was observed on the change of scores of the CDR-SB at week 16 compared with baseline in the two groups $(t=7.34, \mathrm{df}=33$, $\mathrm{p}<0.0001$, and $t=7.89, \mathrm{df}=40, \mathrm{p}<0.0001$ for $\mathrm{OC}$ and LOCF analyses, respectively).

\section{Clinical complications and side effects}

Six side effects were observed over the trial. The difference between the Melissa extract and placebo in the frequency of side effects was not significant except that agitation was less common in the Melissa group (table 2).

\section{DISCUSSION}

This study showed that patients with mild to moderate Alzheimer's disease receiving Melissa officinalis extract experienced significant benefits in cognition after 16 weeks of treatment. The clinical relevance of these findings was emphasised by the improvements seen in both the ADAS-cog and the CDR-SB measures in the Melissa extract group on both "observed case" and "intention to treat" analyses. To the best of our knowledge, this study is the first clinical trial of Melissa officinalis extract in the treatment of Alzheimer's disease, so it is not possible to make comparisons with others trials. However, there is increasing evidence of the possible efficacy of Melissa officinalis and Salvia officinalis in the management of Alzheimer's disease, ${ }^{11}{ }^{12}$ and two recent reports showed that Melissa officinalis has CNS acetylcholine receptor activity and modulates mood and cognitive performance following acute administration, in agreement with our present findings. ${ }^{14} 15$

The side effects associated with Melissa in this study were generally those expected from cholinergic stimulation, and are similar to those reported with cholinesterase inhibitors. ${ }^{6}{ }^{10}$ Although we did not consider agitation to be a side effect, the frequency of agitation was higher in the placebo group, and 
this may indicate an additional advantage of Melissa officinalis in the management of agitated patients with Alzheimer's disease. This is in agreement with the results of a double blind, placebo controlled study that examined the effect of Melissa officinalis essential oil aromatherapy on ratings of agitation and quality of life in 71 patients suffering from severe dementia. ${ }^{19}$

\section{Implications for research}

The limitations of present study, including the small number of patients and a relatively short period of follow up, should be taken into account and indicate the need for further research.

\section{Conclusions}

The results of this study suggest that Melissa officinalis extract has efficacy in the management of mild to moderate Alzheimer's disease. It also has a beneficial effect on the agitation experienced by patients with this disease, and so may well prove to be a novel natural treatment. We feel these results deserved further investigation.

\section{APPENDIX}

\section{The trial group}

Shahin Akhondzadeh (principal investigator and statistical support; clinical neuropsychopharmacologist)

Mohammad Reza Mohammadi (clinical coordinator; psychiatrist)

Maryam Noroozian (trial programmer; neurologist)

Sina Ohadinia (trialist; medical doctor)

Amir Hossein Jamshidi (pharmacognosist)

Mousa Khani (botanist)

\section{ACKNOWLEDGEMENTS}

This study was supported by two grants from National Research Centre of Medical Sciences, Tehran, Iran and Iranian Academic Centre for Education, Culture and Research to SA. We thank Dr Margaret Tejerizo for editing the manuscript.

\section{Authors' affiliations}

S Akhondzadeh, M Noroozian, M Mohammadi, Roozbeh Psychiatric Hospital, Tehran University of Medical Sciences, Tehran, Iran S Ohadinia, A H Jamshidi, M Khani, Institute of Medicinal Plants, Iranian Academic Centre for Education, Culture and Research, Tehran

Competing interests: none declared

\section{REFERENCES}

1 Katzman R. The prevalence and malignancy of Alzheimer's disease. A major killer. Arch Neurol 1976;33:217-18.

2 Evans DA, Funkenstein HH, Albert MS. Prevalence of Alzheimer's disease in a community population of older persons: higher than previously reported. JAMA 1989;262:2551-6.

3 Akhondzadeh S, Noroozian M. Alzheimer's disease: pathophysiology and pharmacotherapy. IDrugs 2002;4:1 167-72.

4 Francis PT, Palmer AM, Snape M, et al. The cholinergic hypothesis of Alzheimer's disease: a review of progress. I Neurol Neurosurg Psychiatry 1999;54:137-47.

5 Bush Al. Therapeutic targets in the biology of Alzheimer's disease. Curr Opin Psychiatry 2001;14:341-8.

6 Bullock R. New drugs for Alzheimer's disease and other dementias. $\mathrm{Br} J$ Psychiatry 2002;180:135-9.

7 Sky AJ, Grossberg GT. The use of psychotropic medication in the management of problem behaviors in the patients with Alzheimer's disease. Med Clin North Am 1994:78:81 1-22.

8 Weinstock $M$. Selectivity of cholinesterase inhibition: clinical implication for the treatment of Alzheimer's disease. CNS Drugs 1999;12:303-7.

9 Giacobini E. Cholinesterase inhibitor therapy stabilizes symptoms of Alzheimer's disease. Alzheimer Dis Assoc Disord 2000;14:S3-1 1.

10 Bullock R. Drug treatment in dementia. Curr Opin Psychiatry 2001;14:349-53.

11 Perry EK, Pikering AT, Wang WW, et al. Medicinal plants and Alzheimer's disease: integrating ethnobotanical and contemporary scientific evidence. Journal of Alternative and Complementary Medicine 1998:4:419-28.

12 Perry EK, Pikering AT, Wang WW, et al. Medicinal plants and Alzheimer's disease: from etnobotany to phytotherapy. J Pharm Pharmacol 1999;51:527-34.

13 Schhultz V, Hansel R, Tyler V. Rational phytotherapy: a physician's guide to herbal medicine. New York: Springer-Verlag; 1998.

14 Wake G, Court J, Pikering A, et al. CNS acetylcholine receptor activity in European medicinal plants traditionally used to improve failing memory. J Ethnopharmacol 2000;69: 105-14.

15 Kennedy DO, Scholey AB, Tildesley NTJ, et al. Modulation of mood and cognitive performance following acute administration of Melissa officinalis (lemon balm). Pharmacol Biochem Behav 2002;72:953-64.

16 McKhann G, Drachman D, Folstein M, et al. Clinical diagnosis of AD: report of NINCDS-ADRDA work group under the auspices of department of health and human services task force on AD. Neurology 1984;34:939-44

17 Mohs RC, Cochen L. Alzheimer's disease assessment scale (ADAS). Psychopharmacol Bull 1988;24:627-8.

18 Schneiders LS. An overview of rating scales used in dementia. Alzheimer Insights 1996;2:1-7.

19 Ballard C, O'Brien J, Reichelt K, et al. Aromatherapy as a safe and effective treatment for management of agitation in severe dementia: the result of a double blind, placebo controlled trial with Melissa. Clin Psychiatry 2002;63:553-8. 